\title{
Force Measurements of a Flapping Wing with Two Angular Degrees of Freedom and Bio-Inspired Kinematics
}

\author{
Christian L. Morrison, Ruben B.R. Vandenheede, Devesh Kumar, Luis P. Bernal, and Carlos Cesnik \\ University of Michigan, Ann Arbor, MI
}

This paper presents preliminary results of an experimental investigation of the aerodynamics of rigid and flexible flapping wings with bio-inspired kinematics. We consider rigid and isotropic flexible wings undergoing two degrees of freedom periodic motions of the flapping angle and the pitch angle. The wing models are thin flat plates with Zimmerman elliptical planform of aspect ratio 7.2. We present comparisons of two bio-inspired kinematics derived from the hawkmoth kinematics along with a purely sinusoidal motion. The experiments are conducted at conditions relevant to biological flight, properly scaled for water tests, which are Reynolds number of 7100 and a reduced frequency of 0.21 based on flow properties at the $75 \%$ span location. The performance of the flapping motions is compared between the differing motions and between flexible and rigid wings. Time resolved phase-averaged force measurements show the development of two force peaks during the flapping cycle for the bio-inspired kinematics and a single peak for the sinusoidal kinematic. These features are not strongly influenced by wing flexibility. Propulsion efficiency is characterized by the measured figure of merit for each case and it is found that flexibility improves performance in terms of both thrust and efficiency compared to rigid wings.

\section{Nomenclature}

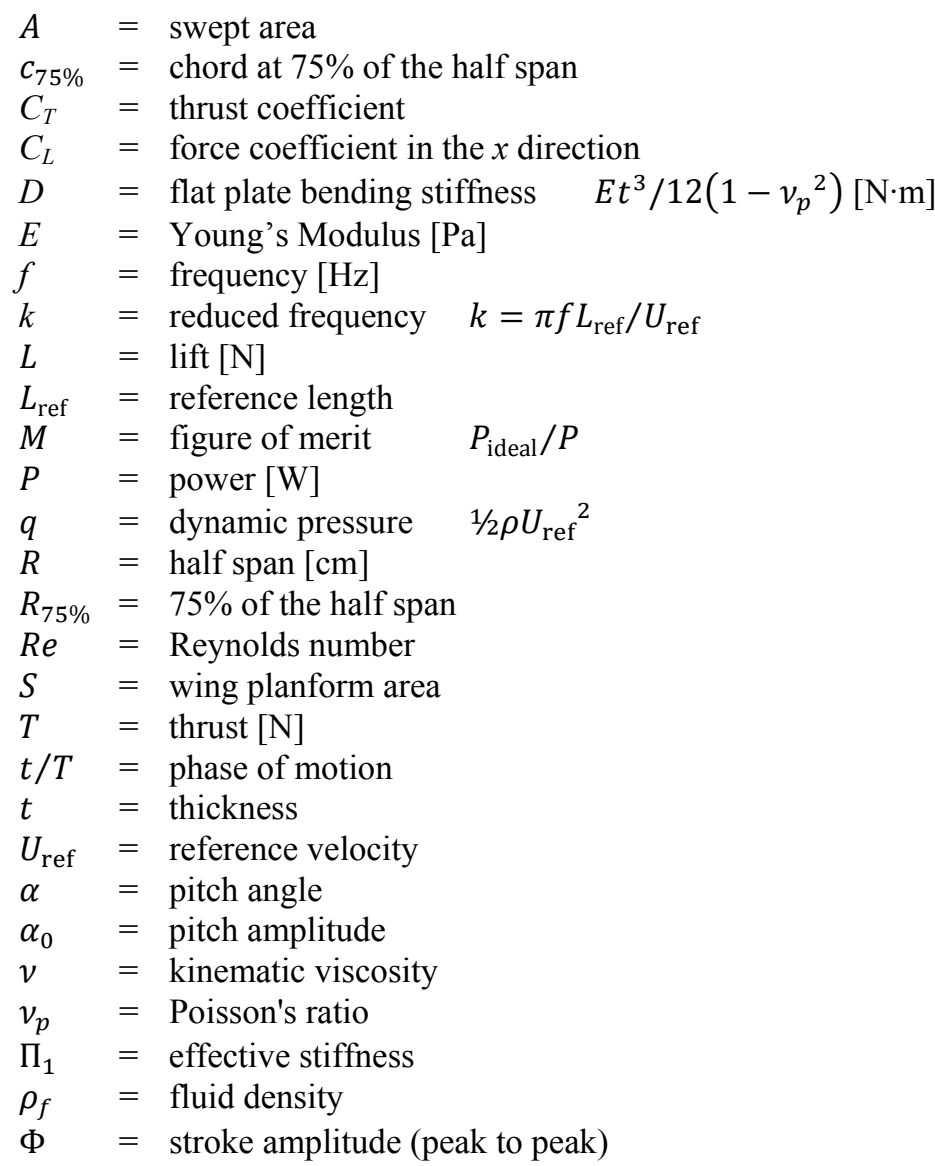


$\phi \quad=$ positional angle of the wing axis in the stroke plane
$\phi_{0}=$ flapping amplitude
\langle\rangle$=$ mean

\section{Introduction}

This paper presents initial results on the aerodynamics of flapping wings motivated by its application to MAV design. Flapping wings have the potential to improve the performance of MAVs in terms of increased maneuverability, control authority, and gust response as compared to fixed wing designs ${ }^{1-2}$. Of particular interest are small birds like hummingbirds and large insects like hawkmoths which show remarkable flight maneuverability and precision suitable for a number of MAV missions. However natural flyers such as the hummingbirds or hawkmoths have complex wing kinematics ${ }^{3}$. A better understanding of the essential features leading to their high aerodynamic performance is needed in order to implement this kinematics in MAV system. Another feature of natural flyers is flexible flapping wings. Several studies ${ }^{1,5}$ have shown that flexible wings can yield better performance when compared to rigid wings over a limited range of the relevant stiffness parameter.

In the present research we extend earlier work on the aerodynamics of pitching and plunging airfoils $\mathrm{s}^{5}$ and wings $\mathrm{s}^{6}$ to flapping motions. A new flapping wing apparatus was developed to study the aerodynamics of wings in hover and forward flight in a water channel. In the current implementation we consider 2-degrees of freedom flapping and pitching motions in hover. We investigate the bio-inspired kinematics of the hawkmoth ${ }^{3}$, first determining the relevant Reynolds numbers and reduced frequencies properly scaled for water tests. In these initial tests we compare the force measurements of these motions to similar pitch-plunge studies in order to determine the role of flapping on aerodynamic performance and propulsion efficiency. Finally, we document the role of flexibly on aerodynamic performance in the context of the bio-inspired kinematics.

\section{Experimental Methods}

In this study, a stepper motor driven assembly is used to drive the wing in an arbitrary periodic motion. The apparatus drives the wing flapping angle, $\phi$, and the pitch incidence angle, $\alpha$, as illustrated in Figure 1. A stepper motor system shown in Figure 2 is installed in a free-surface water channel with test section cross section $60.9 \mathrm{~cm} \mathrm{x}$ $60.9 \mathrm{~cm}$ under quiescent conditions at the University of Michigan. The free surface of the water channel is located 4 $\mathrm{cm}$ above the flapping axis of rotation. To acquire force measurements we utilize a six-component ATI Nano 25 IP68 submersible force/torque transducer and associated instrumentation. The force/torque sensor is located at the root of the wing as shown on the left portion of Figure 2. The force data are recorded with a NI USB-6225 data acquisition board. In order to synchronize the acquired data with the motion of the wing, TTL pulses are generated four times per cycle by the stepper motor drivers. The wing motion is actuated by two Velmex B4800TS series rotary stages through a custom gear train both shown in Figure 2. The test airfoils, which span $241 \mathrm{~mm}$ and a chord of $79 \mathrm{~mm}$, consist of Zimmerman planform wings, in this case two quarter ellipses sharing a major axis at the quarter chord line. Figure 3 is an image of the flexible test article fabricated for rotation about the leading edge of the wing and reinforced with a triangular aluminum plate at the mounting location. 


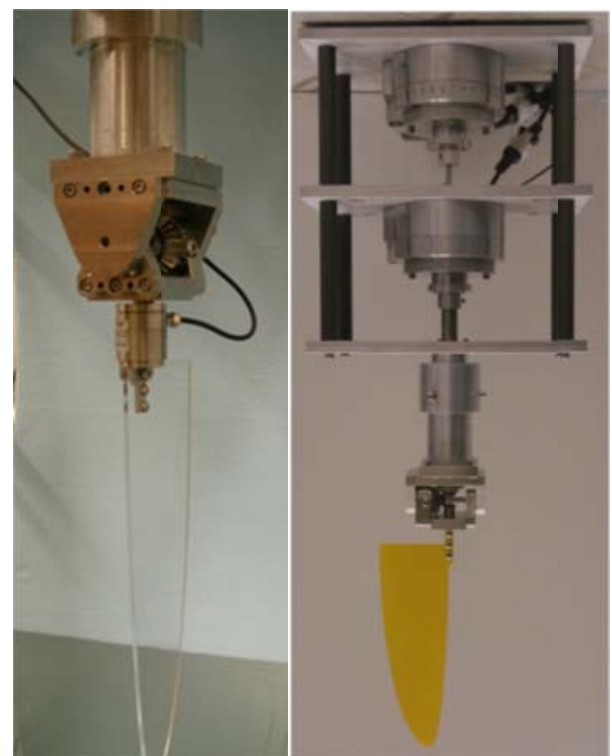

Figure 2. Flapping apparatus and load cell.

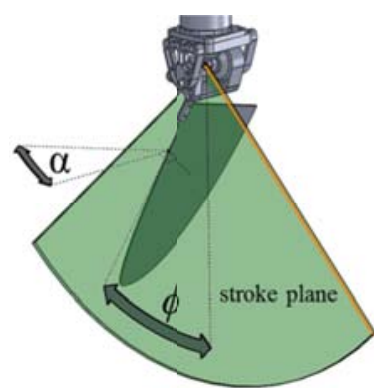

Figure 1. Coordinate system

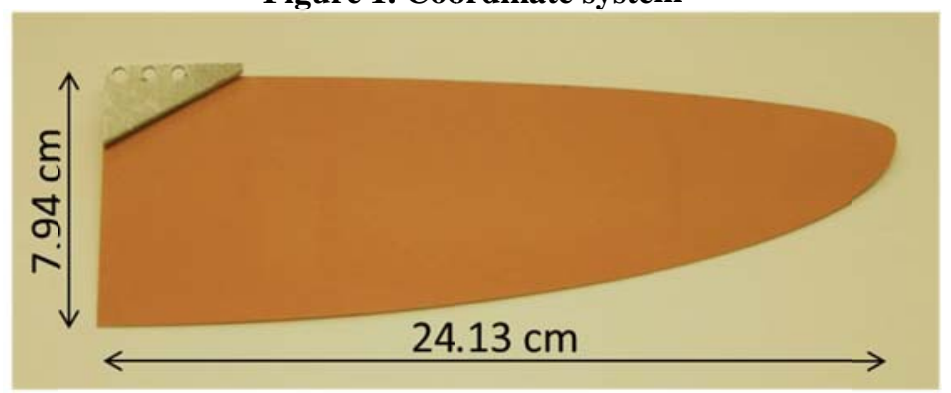

Figure 3. Flexible aluminum wing and support.

The flexible airfoil was water jet cut from sheet aluminum and has a nondimensional stiffness parameter $\Pi_{1}$ of 57. The thickness was chosen because it produces a value of the $\Pi_{1}$ parameter near maximum thrust demonstrated in a previous flexible wing studies. ${ }^{4}$

$$
\begin{aligned}
D & =\frac{E t^{3}}{12\left(1-v^{2}\right)} \\
\Pi_{1} & =\frac{D}{\rho_{f} U_{\mathrm{ref}}^{2} L_{\mathrm{ref}}^{3}}
\end{aligned}
$$

In order to reconcile the forces measured in these experiments with conventional aerodynamic force directions the following definitions are used. The thrust force and the symmetry plane are defined normal to the stroke plane as seen in Figure 1. The lift force is normal to the thrust on the symmetry plane and the side force is in the direction of the cross product, thrust $\times$ lift, which for the configuration shown in figure 1 is directed downward. The flapping angle $\phi$ is the angle between the side force direction and the projection of the pitching axis of rotation of the wing on the stroke plane. A positive flapping angle corresponds to the wing located on the positive side of the lift axis. The pitch angle $\alpha$ is the angle formed by the wing chord and the plane containing the thrust vector and the axis of rotation of the wing. This angle is positive when at flap angle zero the lift is positive for steady flow conditions.

A tare procedure was developed to isolate the hydrodynamic force from other contributions to the force and torque acting on the sensor. These include static tare to remove contributions of the gravity force which changes with flap and pitch angles, and dynamic tare to remove model inertia. The dynamic contributions are removed by repeating the experiments with exactly the same kinematics in air and water. In these experiments the force signals ( 6 channels) are digitized at $2 \mathrm{kHz}$ and low-pass-filtered using a $5^{\text {th }}$ order Butterworth filter with a $2.5 \mathrm{~Hz}$ cut-off frequency (c.f. flapping frequency $0.14 \mathrm{~Hz}$ ). The filtered raw data are converted to physical units using the factory calibration of the sensor. Approximately 100 cycles from a single continuous motion excluding fifteen start-up cycles and the last partial cycle are phase averaged to determine the phase-averaged results. The static tare consists of experiments conducted in air and water using the same motion kinematics but instead of running the motion continuously the apparatus stops at 50 discrete locations and data are measured at each location for two seconds after the movement transient disappears. The static tare data are converted to physical units and interpolated using a 6 -term Fourier series representation of the averaged data at the 50 locations.

The data reported here are phased-averaged tare-corrected results. For both the water and air experiments the static force are subtracted from the dynamic data to remove the static contribution due to model location. Then the 
dynamic tare is performed by subtracting from the water static-corrected results the air static-corrected results. At every data point in this force curve the pitching and flapping angles are known and used to transform measured results in the sensor coordinate system into the laboratory coordinate frame of lift, thrust, side force and corresponding moments.

In order to calculate the work done on the airfoil, it is convenient to work on the sensor frame. The work done by the pitching axis is simply the torque about the z-axis of the sensor multiplied by the pitching rotation rate. The contribution due to the flapping motion is slightly more subtle since the load cell is not located at the center of the flapping rotation. The torque in the flapping plane about the center of the flapping axis is composed of the sum of the torque in the flapping plane about the sensor center and the force in the flapping plane multiplied by the distance of the sensor center to the flapping axis.

\section{Results}

There are two nondimensional parameters for flapping motion in hover, the Reynolds number and the reduced frequency. First we define the Reynolds Number based on the mean velocity of the pitch pivot point at $75 \%$ span, and the wing's chord at that location.

$$
R e=\frac{U_{\mathrm{ref}} L_{\mathrm{ref}}}{v}=\frac{2 \Phi f R_{75 \%} c_{75 \%}}{v}
$$

The unsteady nondimensional parameter to match is the reduced frequency, and it is defined using the same reference parameters.

$$
k=\frac{\pi f L_{\mathrm{ref}}}{U_{\mathrm{ref}}}=\frac{\pi c_{75 \%}}{2 \Phi R_{75 \%}}
$$

We define the resulting dimensional parameters below using the selected Reynolds number of 7100 and a reduced frequency of 0.21 .

Table 1. Experimental parameters.

\begin{tabular}{ccc} 
Parameter & Value & Unit \\
\hline \hline$R e$ & 7100 & - \\
$k$ & 0.21 & - \\
$U_{\text {ref }}$ & 12.2 & $\mathrm{~cm} / \mathrm{s}$ \\
$L_{\text {ref }}$ & 5.8 & $\mathrm{~cm}$ \\
$f$ & 0.14 & $\mathrm{~Hz}$ \\
\hline
\end{tabular}



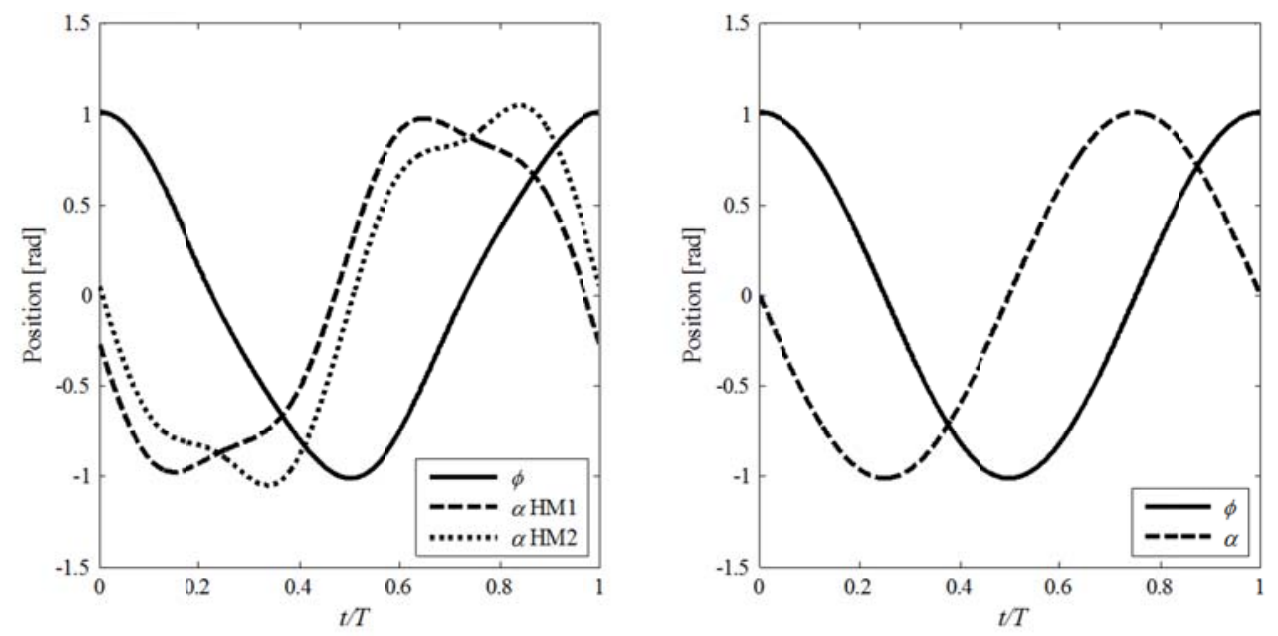

Figure 4. Flapping angle and incidence angle time histories used in the experiments. (a) The bioinspired motions. (b) The sinusoidal motions

The motion histories are selected based on the kinematics of the hawkmoth as measured by Willmott and Ellington. ${ }^{1}$ The flapping wing apparatus has only two degrees of freedom while the wing of the insect moves with three degrees of freedom, defined by the stroke angle, incidence angle and elevation angle. As discussed by Vandenheede at al. ${ }^{6}$ several possibilities exist to implement the hawkmoth kinematic in a two degree of freedom apparatus. Two are used in this study similar to the kinematics used by Vandenheede et $^{2} \mathrm{l}^{6}$. Kinematic HM1 has the same flap angle and incidence angle as the hawkmoth and the hawkmoth elevation angle is ignored. Kinematic HM2 has the same flap angle history, but the pitch angle is corrected to produce the same pitch angle that an observer moving with the wing at the $75 \%$ span would find for the hawkmoth wing motion including the elevation angle. The procedure to calculate this modified pitch angle history is described by Vandenheede et al. ${ }^{6}$ These motion kinematics are plotted in Figure 4. HM1 has maximum/minimum pitch angle earlier in the cycle at $\mathrm{t} / \mathrm{T} \sim 0.65 / 0.15$ and reduced pitch angle rotation rate up to $\mathrm{t} / \mathrm{T} \sim 0.85 / 0.35$. In contrast for the HM2 kinematics the maximum/minimum pitch angle appears later in the cycle at $\mathrm{t} / \mathrm{T} \sim 0.85 / 0.35$, and the slower rotation rate is before the maximum/minimum pitch angle. For reference, experiments were also conducted for a sinusoidal motion of the same amplitude and frequency in both the flapping and pitch angles, which are also shown in Figure 4.
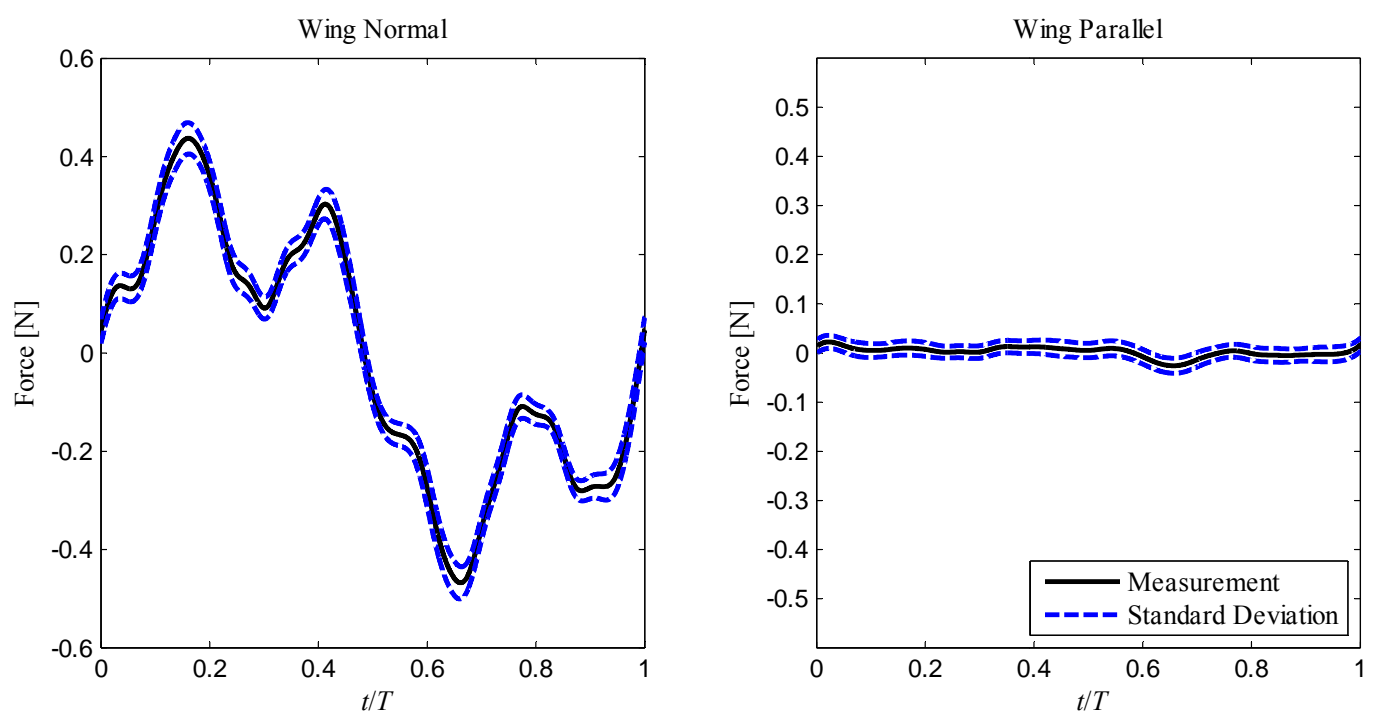

Figure 5. Processed force signal and standard deviation. 
Figure 5 shows typical results of the measured aerodynamic force for the HM2 kinematics. The results plotted are in the sensor coordinate frame. The results show that the wing normal force is much larger than the chordwise as expected for a thin airfoil with sharp leading and trailing edges. Also shown in figure 5 is the measurement uncertainty which is defined as one standard deviation of the ensemble averaged, where the uncertainty of the various tare contributions are combined assuming statistical independence. The typical measurements uncertainty for these results is approximately $7 \%$.

In the results described below the thrust coefficient is defined based on the reference speed at the $75 \%$ span location and wing area. Another important parameter is the propulsion efficiency of flapping wings. Here we use the figure of merit frequently used to characterize propeller and rotor efficiency. The ideal power required to produce a thrust $\mathrm{T}$ is given by equation 5, where $\mathrm{A}$ is the area swept by the flapping wing and $\rho$ is the fluid density. The figure of merit $\mathrm{M}$ is the ratio $\mathrm{M}=\mathrm{P}_{\text {ideal }} / \mathrm{P}_{\phi}$.

$$
P_{\text {ideal }}=\sqrt{\frac{T^{3}}{2 \rho A}}
$$

In order to assess the thrust performance of the bio-inspired kinematics force measurements were taken for HM1, HM2 and the sinusoidal kinematics. The experiments were conducted with a rigid wing and a flexible wing and for parameters as described above. Phase-averaged results are given in Figures 6 and 7 and mean results are summarized in Table 2.
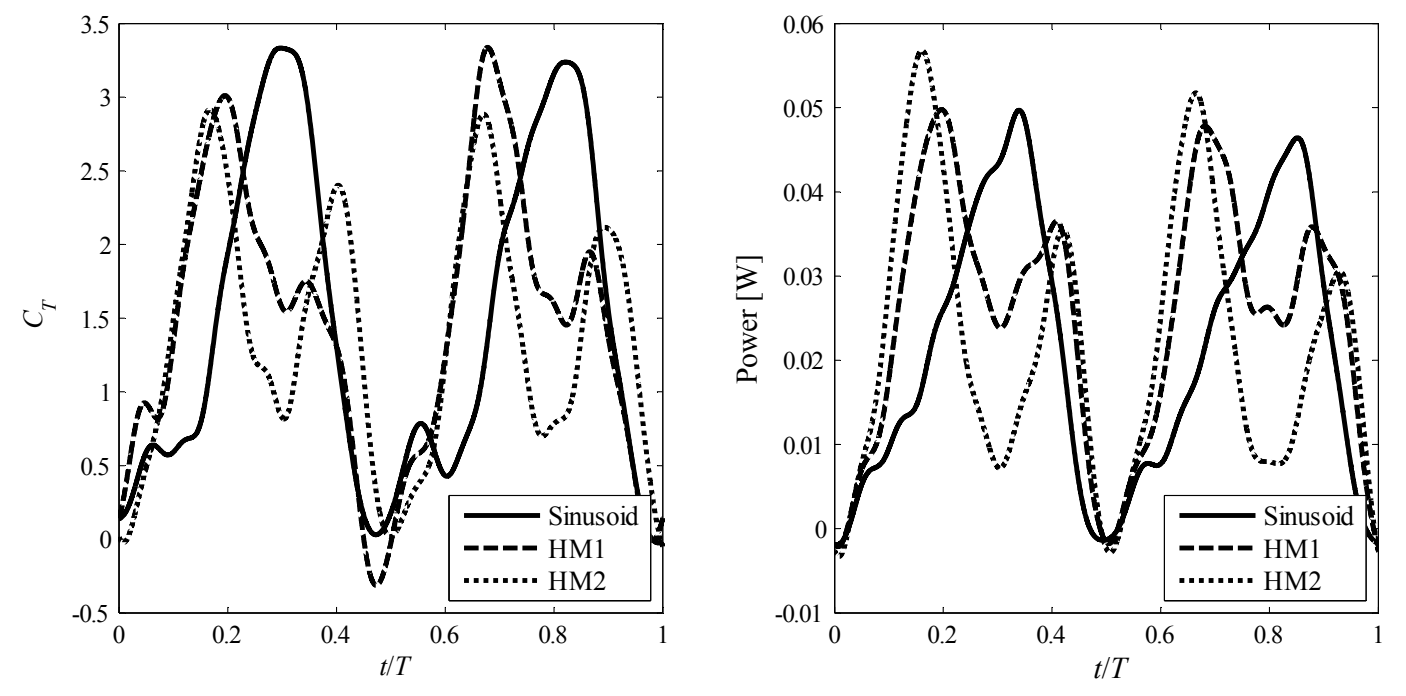

Figure 6. Rigid force and power time histories.

Figure 6 shows the thrust coefficient and input mechanical power for the rigid wing. For the present hover kinematics the thrust and power are expected to be periodic of period $T / 2$ since the up and down stroke should produce substantially the same thrust and require the same input power. However, the measured results show small discrepancies between up and down stroke, which are not well understood. They could be due to installation asymmetries or water tunnel blockage effects. In all cases maximum thrust coefficients around 3 are measured. These values are consistent with comparable results for pitch-plunge motions reported by Vandenheede $a t a l .{ }^{6}$ The sinusoidal kinematics show an early peak in thrust coefficient at $\mathrm{t} / \mathrm{T} \sim 0.1$ followed by a larger peak at $\mathrm{t} / \mathrm{T} \sim 0.25$. For the HM1 kinematics there are three peaks in thrust coefficient at $t / T \sim 0.1,0.2,0.35$. For the HM2 kinematics the peak are at $\mathrm{t} / \mathrm{T} \sim 0.15$ and 0.4. Input power results follow the thrust coefficient results and input power generally follows thrust coefficient amplitude except for the first peak at $\mathrm{t} / \mathrm{T} \sim 0.1$ which does not show comparable maxima in input power for the sinusoidal and HM1 kinematics. The input power peak at $\mathrm{t} / \mathrm{T} \sim 0.4$ for the HM1 and HM2 kinematics is more pronounced than the thrust coefficient peak. It is clear that small changes in pitch angle history 
produce large changes in the thrust coefficients and input power, and a better understanding of the underlying flow development is necessary to fully account for these changes.
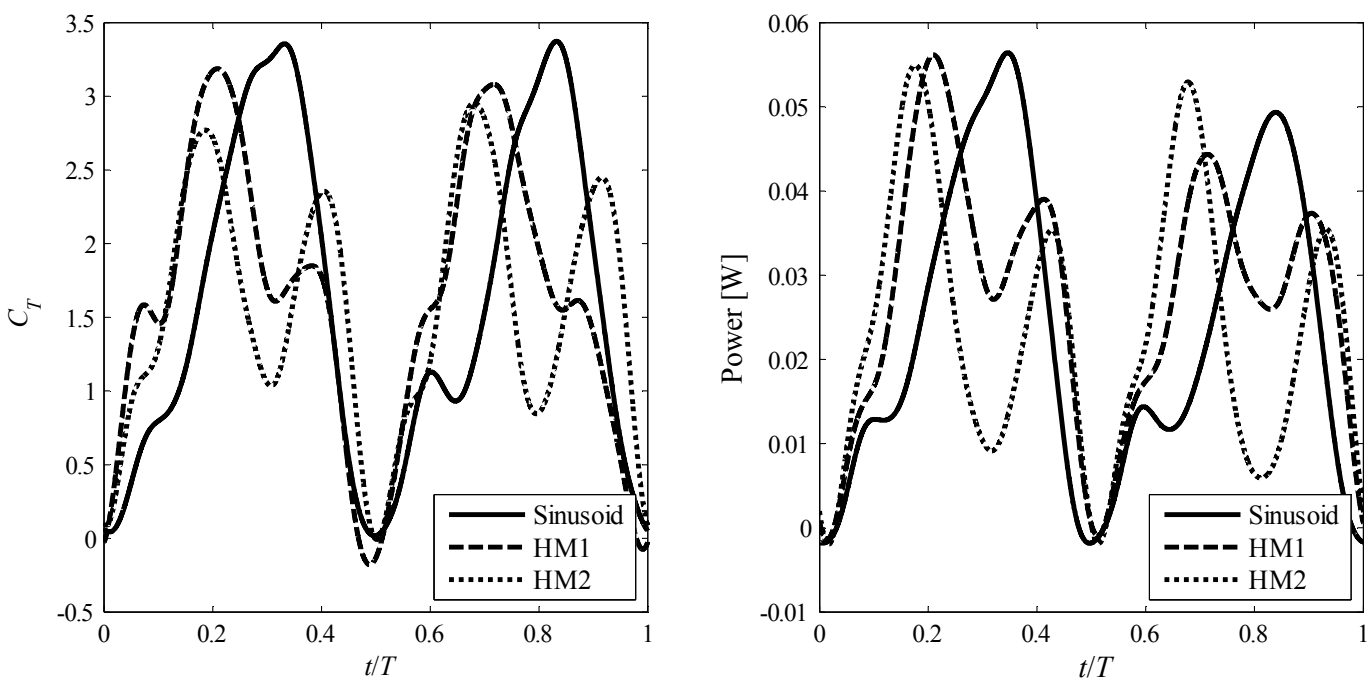

Figure 7 Flexible force and power time histories.

Figure 7 shows the results for the flexible wing using the same kinematics as for the data in figure 6 for a rigid wing. The main feature of the thrust coefficient and input power histories remain the same. There are small changes in amplitude with the flexible wing producing larger thrust coefficient and input power than the corresponding rigid wing.

Cycle means and propulsion efficiency results are summarized in Table 2. Clearly the flexible wing produces larger mean thrust coefficients for all cases considered. In contrast motion kinematics does not seem to impact mean thrust very much despite the large changes in force history. Figure of merit results in table 2 show that HM1 and HM2 kinematics for the flexible wing are significantly improved compared to the corresponding rigid wing.

Table 2. Cycle averaged performance of the kinematics for the rigid and flexible wing.

\begin{tabular}{cccccccc} 
& Motion & $\langle\boldsymbol{T}\rangle[\mathbf{N}]$ & $\left\langle\boldsymbol{C}_{\boldsymbol{T}}\right\rangle$ & $\langle\boldsymbol{P}\rangle[\mathbf{m W}]$ & $\left\langle\boldsymbol{P}_{\boldsymbol{\phi}}\right\rangle[\mathbf{m W}]$ & $\boldsymbol{P}_{\text {ideal }}[\mathbf{m W}]$ & $\boldsymbol{M}$ \\
\hline \hline \multirow{4}{*}{ Rigid } & sin & 0.167 & 1.47 & 21.2 & 20.5 & 5.17 & 0.244 \\
& HM1 & 0.167 & 1.47 & 25.2 & 23.4 & 5.17 & 0.205 \\
& HM2 & 0.156 & 1.38 & 21.8 & 21.3 & 4.68 & 0.215 \\
\hline \multirow{4}{*}{ Flex } & sin & 0.181 & 1.60 & 23.8 & 22.9 & 5.87 & 0.247 \\
& HM1 & 0.184 & 1.63 & 27.3 & 25.1 & 6.02 & 0.220 \\
& HM2 & 0.178 & 1.57 & 23.5 & 22.7 & 5.70 & 0.243 \\
\hline
\end{tabular}

\section{Conclusion}

Using a two degree of freedom flapping apparatus, we have measured the fluid forces on a rigid and a flexible wing undergoing bio-inspired motions. We selected the stiffness of the flexible wing to yield a significant performance change based on a nondimensional analysis. While we see that the kinematics measured in this analysis do not perform significantly better than pure sinusoidal motion, the bio-inspired kinematics demonstrate drastically different force time histories. Furthermore the flexible wing airfoils exhibit a slightly increased performance when compared to rigid wing airfoils under the same motion conditions. 
The bio-inspired kinematics do not have better thrust or performance than the purely sinusoidal motion, but the hover motion with the incidence angle corrected for the elevation angle shows a significant improvement over the motion neglecting the effect of the elevation angle entirely.

The three primary results of this work are that the bio-inspired motions produce two distinct force peaks during the half stroke while the sinusoidal motion produces a single peak. The sinusoidal flapping kinematic produces the larges thrust coefficient and performance for the cases studied. Finally, the isotropic flexible wings produced slightly more thrust and performance for identical kinematics using a rigid wing. Comparison with pitch-plunge motion $^{6}$, the present flapping wing results show comparable thrust time histories with the pitch-plunge cases showing slightly lower thrust coefficient, which can be attributed to normalization.

\section{Acknowledgement}

The research was sponsored by the Air Force Office of Scientific Research's Multidisciplinary University Research Initiative (MURI) on Biologically-Inspired Anisotropic Flexible Wing for Optimal Flapping Flight and by the Michigan/AFRL (Air Force Research Laboratory) Collaborative Center in Aeronautical Sciences.

\section{References}

${ }^{1}$ Shyy W., Lian Y., Tang J., Viieru D., and Liu H. Aerodynamics of low Reynolds number flyers. Cambridge University Press, 2008.

${ }^{2}$ Shyy W., Aono H., Chimakurthi S. K., Trizila P., Kang C. K., Cesnik C.E.S., and H. Liu. Recent progress in flapping wing aerodynamics and aeroelasticity. Progress in Aerospace Sciences, 46:284-327, 2010.

${ }^{3}$ Willmott, A. P. and Ellington, C. P., "The mechanics of flight in the hawkmoth Manduca Sexta. I. Kinematics of hovering and forward flight," Journal of Experimental Biology 200, pp. 2705-22.

${ }^{4}$ Rausch, J. M., Bernal, L. P., Cesnik, C. S., Shyy, W., Ukeiley L., "Fluid Dynamic Forces on Plunging Spanwise-Flexible Elliptical Flat Plates at Low Reynolds Numbers," AIAA-2011-3435.

${ }^{5}$ Baik Y., Rausch J., Bernal L. P., and Ol M. V. "Experimental investigation of pitching and plunging airfoils at reynolds number between $1 \times 10^{4}$ and $6 \times 10^{4}$ ". AIAA-2009-4030.

${ }^{6}$ Vandenheede R., Bernal L. P., Morrison C. L., Humbert S., "Force Generation of Bio-inspired Hover Kinematics," AIAA2012-0708. 